\title{
Thin Film Analysis by Nanomechanical Infrared Spectroscopy
}

Casci Ceccacci, Andrea; Cagliani, Alberto; Marizza, Paolo; Schmid, Silvan; Boisen, Anja

Published in:
ACS Omega

Link to article, DOI:

10.1021/acsomega.9b00276

Publication date:

2019

Document Version

Publisher's PDF, also known as Version of record

Link back to DTU Orbit

Citation (APA):

Casci Ceccacci, A., Cagliani, A., Marizza, P., Schmid, S., \& Boisen, A. (2019). Thin Film Analysis by Nanomechanical Infrared Spectroscopy. ACS Omega, 4(4), 7628-7635.

https://doi.org/10.1021/acsomega.9b00276

\section{General rights}

Copyright and moral rights for the publications made accessible in the public portal are retained by the authors and/or other copyright owners and it is a condition of accessing publications that users recognise and abide by the legal requirements associated with these rights.

- Users may download and print one copy of any publication from the public portal for the purpose of private study or research.

- You may not further distribute the material or use it for any profit-making activity or commercial gain

- You may freely distribute the URL identifying the publication in the public portal

If you believe that this document breaches copyright please contact us providing details, and we will remove access to the work immediately and investigate your claim. 


\title{
Thin Film Analysis by Nanomechanical Infrared Spectroscopy
}

\author{
Andrea Casci Ceccacci, ${ }^{*}{ }^{\dagger}$ Alberto Cagliani, ${ }^{\dagger}$ Paolo Marizza, ${ }^{\dagger}$ Silvan Schmid, ${ }^{\ddagger}$ and Anja Boisen ${ }^{\dagger}$ \\ ${ }^{\dagger}$ Department of Micro- and Nanotechnology, Technical University of Denmark, 2800 Kongens Lyngby, Denmark \\ ${ }^{\sharp}$ Institute of Sensor and Actuator Systems, TU Wien, 1040 Vienna, Austria
}

Supporting Information
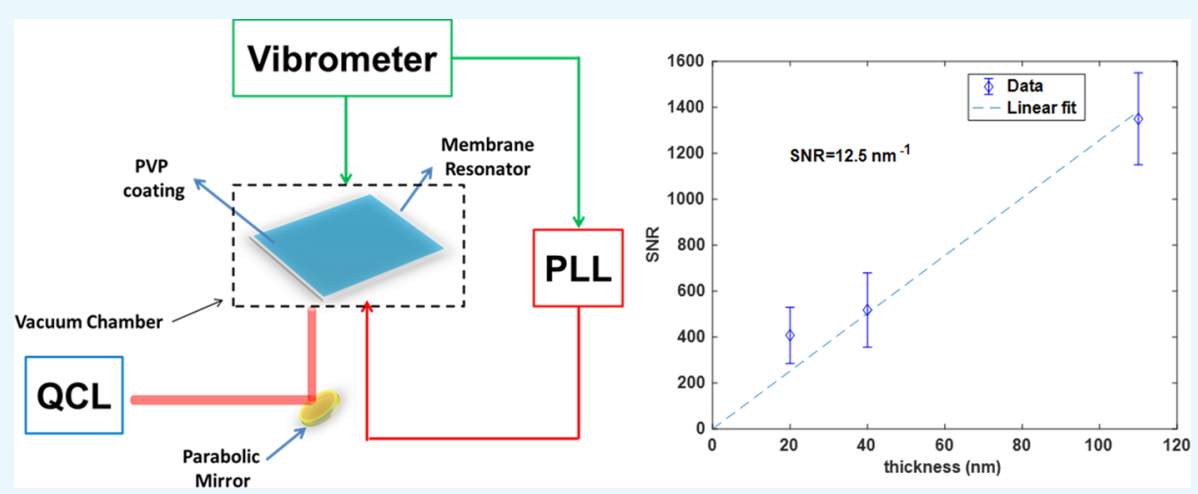

ABSTRACT: There is a fundamental need for techniques for thin film characterization. The current options for obtaining infrared (IR) spectra typically suffer from low signal-to-noise-ratios (SNRs) for sample thicknesses confined to a few nanometers. We present nanomechanical infrared spectroscopy (NAM-IR), which enables the measurement of a complete infrared fingerprint of a polyvinylpyrrolidone (PVP) layer as thin as $20 \mathrm{~nm}$ with an SNR of 307. Based on the characterization of the given NAM-IR setup, a minimum film thickness of only $160 \mathrm{pm}$ of PVP can be analyzed with an SNR of 2 . Compared to a conventional attenuated total reflectance Fourier transform infrared spectroscopy (ATR-FTIR) system, NAM-IR yields an SNR that is 43 times larger for a $20 \mathrm{~nm}$-thick PVP layer and requires only a fraction of the acquisition time. These results pave the way for NAM-IR as a highly sensitive, fast, and practical tool for IR analysis of polymer thin films.

\section{INTRODUCTION}

The ability to engineer the chemical and physical properties of surfaces using dedicated functionalization methods is of fundamental importance for the advancement in many technological fields, such as bio and chemical sensing, ${ }^{1}$ drug delivery, ${ }^{2}$ tissue engineering, ${ }^{3}$ solar cells, ${ }^{4}$ thin films batteries, ${ }^{5}$ and fabrication of semiconductor devices. ${ }^{6}$ In recent years, the possibility of engineering the chemical and physical properties of a surface by applying a thin polymer film has attracted considerable attention due to its simplicity, robustness, and flexibility, due to the vast selection of polymer films. ${ }^{2,3,7-9}$ Thin polymer films can exhibit confinement-induced, peculiar physicochemical properties, such as changes in glass transition temperature, ${ }^{10}$ elastic moduli, ${ }^{11}$ and crystallization. ${ }^{12}$ Characterizing the properties of such thin films is therefore of high relevance both technologically and scientifically.

The chemical behavior and structure of thin films are often characterized by infrared absorption spectroscopy ${ }^{13-16}$ or other vibrational spectroscopy techniques such as surfaceenhanced Raman Spectroscopy. ${ }^{17}$ Thin film IR spectroscopy is commonly performed using attenuated total reflectance Fourier transform infrared spectroscopy (ATR-FTIR), with a typical configuration with only one reflection within the ATR crystal. ${ }^{16,18,19}$ An issue connected with the use of ATR-FTIR is that the signal-to-noise-ratio (SNR) degrades quickly when the sample thickness is much smaller than the penetration depth of the evanescent IR wave of up to $2 \mu \mathrm{m},{ }^{13}$ thereby effectively limiting the thickness range that can be studied. One possibility to overcome this issue is to use very long acquisition times, ${ }^{16}$ but this restricts the throughput of the analytical system. A multireflection ATR accessory can also be used to boost the signal, but such an approach is suitable only when the crystal is in contact with a liquid or the thin functionalization layer to be studied is irreversibly attached to the crystal. These drawbacks might represent an issue in terms of the experimental throughput of ATR-FTIR when a large variety of thin polymer layers with high spectroscopically precisions have to be tested. Hence, there is a need for a fast, reliable, and nondestructive method to analyze thin films.

Infrared reflection-absorption spectroscopy (IRRAS) ${ }^{20}$ is used to study the thin films and needs to be performed on a reflective substrate. ${ }^{21}$ Due to the surface selection rule, the phase of the reflected beam is shifted by an amount that depends on the incident angle, polarization, and wavelength of the incident light. The resulting spectra contain information on the molecules' dipole moment perpendicular to the sur-

Received: January 30, 2019

Accepted: April 8, 2019

Published: April 26, 2019 
face. $^{21-23}$ Furthermore, studies have demonstrated that engineered substrates equipped with antenna-like structures can strongly enhance the light-matter interaction. These substrates have been used to monitor nanoparticles and protein interactions. ${ }^{23}$ Although these approaches have pushed the limit of detection up to a single-molecule layer, the experimental time frame is extended, and it can take up to several hours due to the low SNR on a single acquisition.

Polarization modulation-IRRAS (PM-IRRAS) can overcome some of the limitations of IRRAS by improving the low SNR; however, the spectra are still dependent on the surface selection rules, and the spectra require a complex postprocessing technique. $16,24,25$

The combination of atomic force microscopy (AFM) and infrared spectroscopy has emerged as a hybrid technique known as AFM-IR, which has shown to provide a chemical mapping of submicron polymer films. ${ }^{26-30}$ Although the method can provide accurate spectra and overcome the spatial resolution issue, the experimental setup needs a fine alignment of the light sources with the AFM tip, which might limit the throughput of method and extend the time frames.

Photothermal spectroscopy methods are based on the transduction of the temperature change of the sample, induced by the light absorption, into a signal that can be recorded. Typically, density, pressure, or temperature changes, induced by the wavelength specific light absorption, have been exploited to obtain an IR absorption fingerprint of a sample. $^{31-34}$

In the last 15 years, nanomechanical sensing has been exploited for biosensing purposes ${ }^{35-39}$ and material characterization. $^{40,41}$ In particular, photothermal nanomechanical IR sensing (NAM-IR) has been developed for both deflection and resonance frequency detection schemes. $32,33,42-49$

In the present work, we have used the resonance frequency detection scheme.

During the experiment, the membrane oscillates at its mechanical natural frequency via the actuation provided by an external piezoelectric crystal. The mechanical resonance frequency of the resonator is a function of the mechanical stress, which, in turn, depends on the local temperature. Changes in temperature, occurring upon light absorption, detune the resonance frequency of the resonator. Due to the photothermal effect, ${ }^{31}$ the absorbed light locally increases the temperature, which releases a part of the native stress in the membrane, causing the resonance frequency to drop. ${ }^{50}$ The changes in the resonance frequency are recorded through a lock-in detection scheme and a typical experimental setup as illustrated in Figure 1.The resonance frequency shifts as a function of the wavelength specific light absorption represents the NAM-IR spectra.

Regardless of the detection scheme used, the samples examined so far using NAM-IR have been limited to airbornedeposited materials or liquids. This limitation is due to the lack of mechanical robustness of previous nanomechanical transducers, such as nanostrings ${ }^{45}$ and microcantilevers. ${ }^{47}$ Moreover, such nanomechanical structures suffer from AFM-like readout laser alignment issues due to their micrometer-range lateral dimensions, which can drastically limit the final throughput of NAM-IR. Recently, a filter-like nanomechanical structure $^{51}$ has been developed to improve the sampling efficiency of airborne particles. However, the perforated geometry is not suitable for analyzing thin films. A main advantage of the photothermal spectroscopy is that it does not
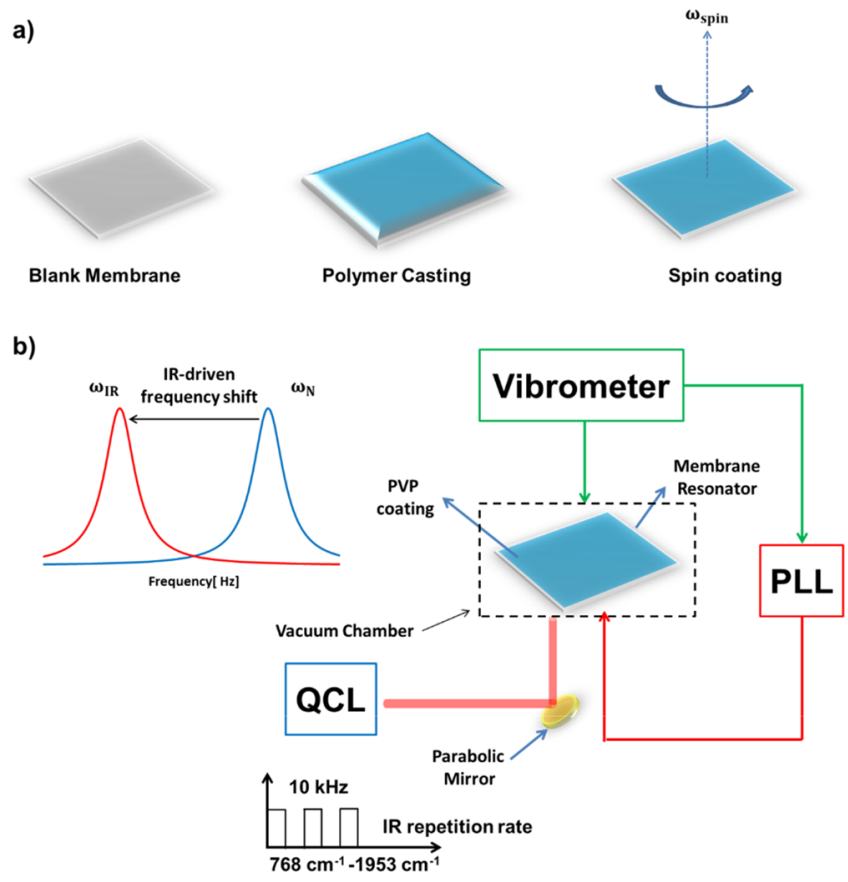

Figure 1. Sampling procedure and NAM-IR setup. (a) Polymer deposition method; (b) NAM-IR spectrometer comprises: a pressurecontrolled chamber providing an optical access from both sides of the sensor, a quantum cascade laser (QCL, Block Engineering) that generates a monochromatic IR light in the range from 768-1953 $\mathrm{cm}^{-1}$, an optical readout system based on a laser Doppler vibrometer (Polytech MSA-500), a phase lock-in amplifier, and a piezo driver. ${ }^{44,45}$

suffer from the typical spectral artefacts of ATR-FTIR or absorbance-reflection techniques in the recorded spectra. ${ }^{13}$ Moreover, it is insensitive to the IR light angle or polarization.

Our NAM-IR system used in this work consists of a thin $(100 \mathrm{~nm})$ square $(1 \times 1 \mathrm{~mm})$ prestressed $(250 \mathrm{MPa})$ siliconrich silicon Nitride (SRN) membrane fabricated by standard silicon micromachining techniques.

Membrane-like resonators have, intuitively, higher mechanical robustness and uniform topography compared to silicon nitride string resonators. Furthermore, membranes withstand typical surface functionalization treatments in liquid, including spray coating and spin coating. As a demonstrator of the capabilities of our NAM-IR system, we analyze polyvinylpyrrolidone (PVP) thin films with thicknesses down to $20 \mathrm{~nm}$. PVP polymer thin films have recently attracted much attention due to their high versatility in drug delivery devices ${ }^{52}$ and hydrogels, ${ }^{53}$ where their physical and chemical properties are crucial. The low thermal mass of our resonating membranes allows running analysis over a range of $1100 \mathrm{~cm}^{-1}$ wavenumbers in $30 \mathrm{~s}$ with an SNR of up to 307, even for a polymer film as thin as $20 \mathrm{~nm}$. In Figure 1, our NAM-IR system is illustrated. It is composed of a vacuum chamber with two apertures, one for the IR light and one for the readout laser of the vibrometer. A quantum cascade laser (QCL) is used to generate the stepwise monochromatic IR light in the range of $768-1953 \mathrm{~cm}^{-1}$ with a step of $0.5 \mathrm{~cm}^{-1}$.

\section{RESULTS AND DISCUSSIONS}

In Figure 2, the NAM-IR spectra of the PVP thin films are compared to those of the conventional spectroscopy techniques. The three mid-IR bands of PVP recorded by NAM-IR match well the spectra recorded by the reference 


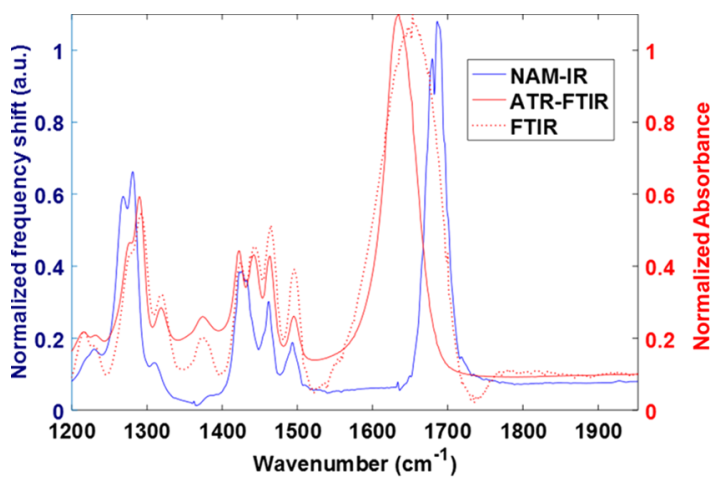

Figure 2. Comparison of the NAM-IR spectrum of a PVP film with respect to ATR and FTIR. The three main bands characterize the polymer fingerprint: the signal at $1300 \mathrm{~cm}^{-1}$ referred to the $\mathrm{C}-\mathrm{N}$ bond stretching and the $\mathrm{CH}_{2}$ ring wag mode. The band with three peaks between 1400 and $1500 \mathrm{~cm}^{-1}$ corresponds to the $\mathrm{CH}_{2}$ ring wag and the $\mathrm{C}-\mathrm{N}$ stretching. The peak at $1680 \mathrm{~cm}^{-1}$ corresponds to the $\mathrm{C}=\mathrm{O}$ stretching mode. The spectra of a $500 \mathrm{~nm}$-thick PVP sample are recorded.

methods (ATR-FTIR and transmission FTIR) as well as the previously published results. ${ }^{54}$ The PVP carbonyl band appears to be shifted toward a higher frequency when recorded through NAM-IR compared to the reference techniques such as FTIR or ATR-FTIR. Photothermal spectroscopy provides a complementary $^{46,55,56}$ technique to probe IR fingerprints of chemical bonds. Absorption spectroscopy relies on the Beer-
Lambert law. Instead, NAM-IR relies on the nonradiative decay, which is a complementary phenomenenon. ${ }^{56}$ Furthermore, the distortion of the refractive index occurring upon absorption $^{25}$ can bias a measured peak toward a lower frequency; ${ }^{25,57}$ such phenomena can be even more pronounced when the sample is a submicron film. ${ }^{13}$ Although correction algorithms can adjust the relative peak intensities as well as the peak positions, ${ }^{25,57}$ such methods require an a priori knowledge on the optical properties of the film sample.

In order to maximize the IR spectrum signal-to-noise-ratio (SNR) of our photothermal spectrometer, we looked closely at the relation between the thermal time constant of the membrane resonator, the minimum settling time of the IR source for each wavelength, and the optimal signal integration time.

First, the time transient of the resonance frequency shift of the membrane sensor upon periodic IR irradiation at a fixed IR wavelength was characterized, in order to extract the thermal time constant of the membrane $\tau_{\mathrm{M}}{ }^{58}$ As can be seen in Figure $3 \mathrm{a}$, the mechanical resonance frequency of the membranes decreases following a first-order exponential decay upon IR absorption. Such an ON-OFF (or heating and cooling) curve has been acquired 485 times, and each of them was fitted with an exponential decay function, $\delta_{\mathrm{f}}(t)=\Delta \mathrm{f}\left(1-\mathrm{e}^{-t / \tau_{\mathrm{M}}}\right)$, extracting the corresponding $\tau_{\mathrm{M}}$. The transient was evaluated for two different vacuum pressures, which yielded (for a $20 \mathrm{~nm}$ PVPcoated membrane) $\tau_{\mathrm{M}}=4.1 \pm 0.08 \mathrm{~ms}$ at a low vacuum of 1 mbar and $\tau_{M}=17 \pm 0.9 \mathrm{~ms}$ at a high vacuum of $10^{-5} \mathrm{mbar}$.

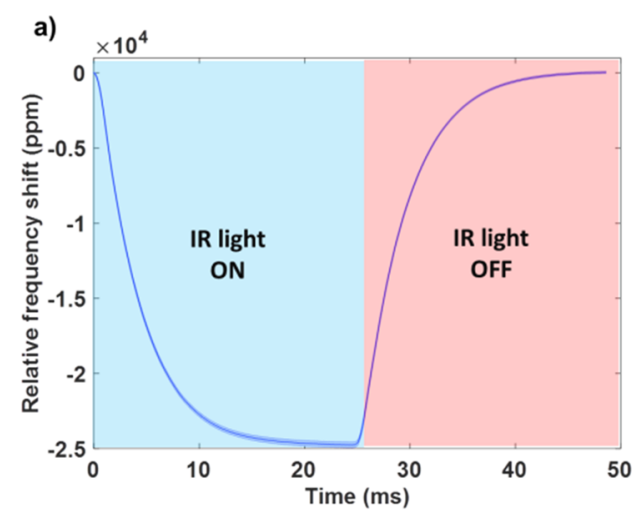

b)
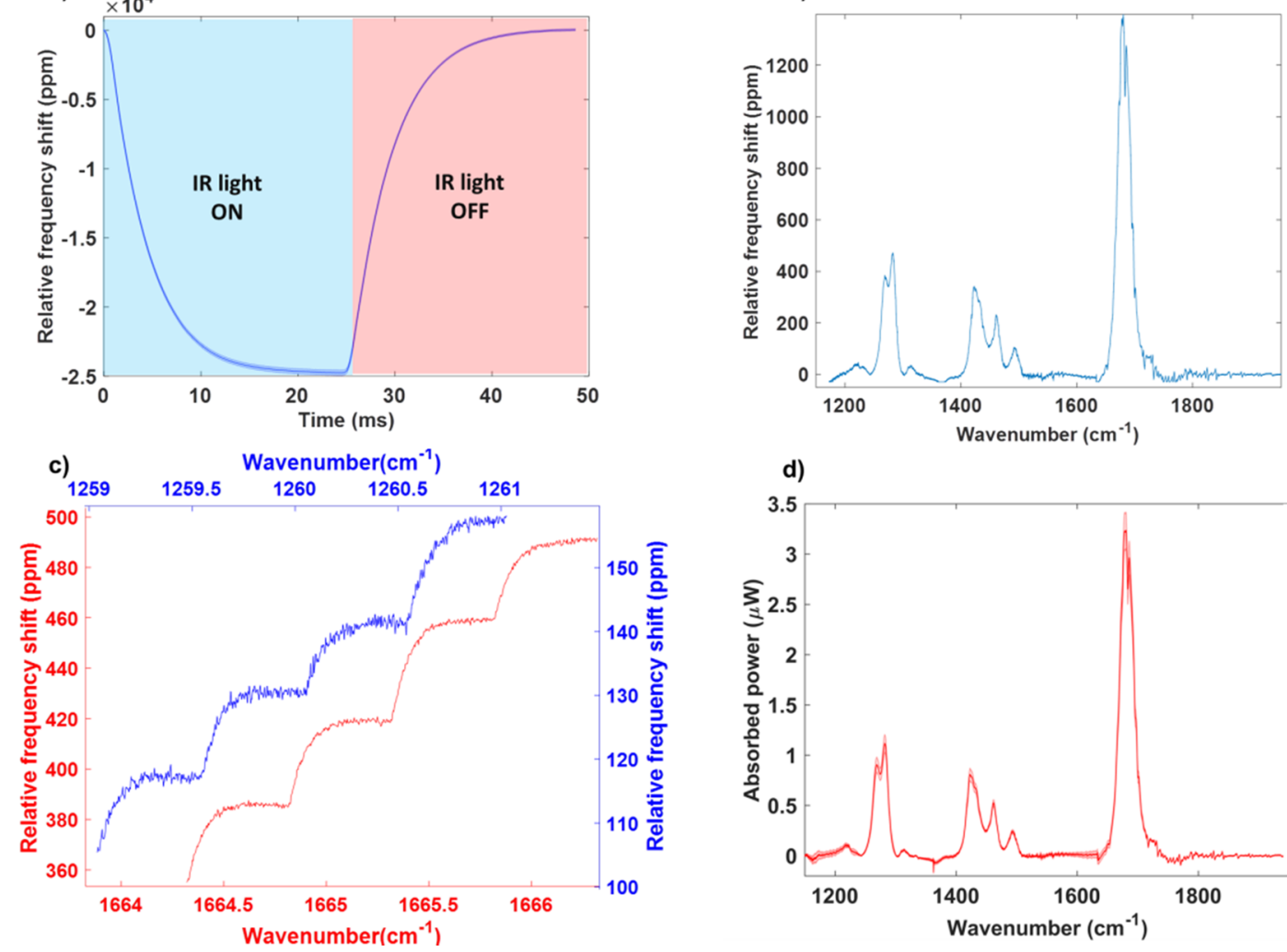

d)

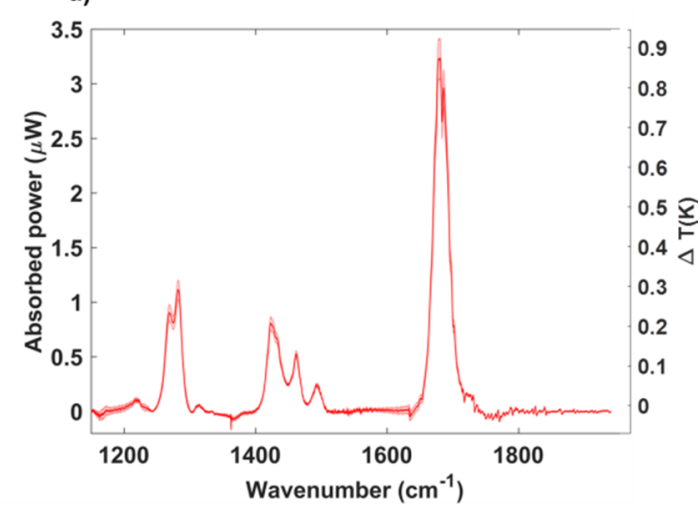

Figure 3. (a) Relative frequency shift upon periodic light absorption. The values are recorded on a $200 \mathrm{~nm}$ coated membrane at 1 mbar; the shades represent the standard deviation of 485 overlapped ON-OFF curves. (b) Raw NAM-IR spectra. (c) Membrane NAM-IR spectrophotometer can recognize the QCL steps of $0.5 \mathrm{~cm}^{-1}$. (d) Absorbed power throughout the wavelength range and relative temperature reached during a sweep on a $20 \mathrm{~nm}$ PVP-coated membrane in HV. On the right-hand vertical axis is the relative temperature shift established in the membrane upon absorption. As a reference value, the baseline temperature was considered as $293.15 \mathrm{~K}$. 
Due to the finite thermal mass of the sensor, ${ }^{42,58}$ the thermal time constant upon light absorption (heating) is higher than the cooling time constant. Instead, the difference in the $\tau_{\mathrm{M}}$ value is attributed to the different relative importance of the heat transport mechanism involved at the two vacuum levels. In high vacuum and at room temperature, the mean free path of the gas molecules becomes roughly $10 \mathrm{~m}$, which is significantly larger than the critical dimension of the nanomechanical resonator. Hence, the heat transport due to natural convection can be neglected, and all the heat generated in the membrane is dissipated by conduction through the body chip alone. Contrary, at $1 \mathrm{mbar}$, the mean free path corresponds to roughly $100 \mu \mathrm{m}$, which is comparable to the dimensions of the device, hence, allowing a part of the heat to be dissipated by conduction through the surrounding gas. Therefore, the two regimes have different time transient responses. The response time is also influenced by the thickness of the polymer layer, and the corresponding values are reported in the Supporting Information. A precise knowledge of the thermal time constant $\tau_{\mathrm{M}}$ is required in order to choose an optimal settling time for each wavelength emitted by the QCL. The settling time was set such that the membrane would be exposed for no less than $3 \times \tau_{M}$ for each discrete IR wavelength, in order to ensure that the membrane reaches the maximum frequency shift at each wavelength step. Considering that the QCL sweeps $1185 \mathrm{~cm}^{-1}$ with steps of $0.5 \mathrm{~cm}^{-1}$, the total acquisition time for a $20 \mathrm{~nm}$ thin polymer layer is only $28.4 \mathrm{~s}$, given a $\tau_{\mathrm{M}}$ value of $\sim 4 \mathrm{~ms}$ in low vacuum.

The membranes' transient thermal constant also influences the choice of the integration time of the phase-locked loop (PLL) detection scheme. To correctly follow the resonance frequency shift upon light absorption for each IR wavelength step, the lock-in amplifier bandwidth has to be set sufficiently high enough to follow the full dynamics of the resonance frequency shift determined by $\tau_{\mathrm{M}}$, but at the same time, a low bandwidth is desirable to reduce the frequency acquisition noise. The best balance was found by measuring the Allan deviation of the frequency signal as function of the PLL integration time ${ }^{59}$ (see the Supporting Information).

Figure $3 b, c$ shows the raw data acquired by our setup. The detailed understanding of the membrane frequency behavior enabled us to clearly resolve each distinct IR wavelength step of $0.5 \mathrm{~cm}^{-1}$, since the exponential thermal response of the membrane frequency is clearly visible, and the signal arrives at the maximum amplitude within each step.

In this work, the integration time was set to $1.5 \mathrm{~ms}$ for low vacuum experiments and $7 \mathrm{~ms}$ for high vacuum experiments, leading to a resolvable frequency shift of $2 \mathrm{ppm}$ for a membrane coated with a $20 \mathrm{~nm}$-thin PVP layer.

Since different polymers, ${ }^{60,61}$ drugs, ${ }^{62}$ brush layers, ${ }^{63}$ and block coplymers ${ }^{64}$ can typically present phase transition temperatures around room temperature, it is of the highest importance to ensure the thermal stability of the sample properties during the IR illumination while the spectrum is recorded. Therefore, for NAM-IR to be a viable solution for ultrathin organic samples, the increase in sample temperature during the spectrum acquisition needs to be minimized. By maximizing the SNR of the overall system, we could minimize the necessary IR power and therefore achieved a spectrum with an SNR > 200 for a change in the sample temperature below 1 $K$. Despite the importance of the issue, there has, to the best of our knowledge, been no previously presented any estimation of the temperature rise due to the IR absorption for NAM-IR systems. We used an analytical model to estimate the power absorbed by the membranes. ${ }^{51}$ Considering a frequency noise floor of $2 \mathrm{ppm}$ (see the Supporting Information), the minimum power that can be detected is $5 \mathrm{nW}$ for an integration time of $7 \mathrm{~ms}$ and an SNR of 1 . Such an absorbed power corresponds to a local temperature increase in the membrane center of $3 \mathrm{mK}$ for a $20 \mathrm{~nm}$ PVP-coated membrane (see the Supporting Information). In the case of a $20 \mathrm{~nm}$ coated membrane, the maximum absorbed power is $3.5 \mu \mathrm{W}$ (at $1680 \mathrm{~cm}^{-1}$ ), which is about $1 \%$ of the incident QCL laser power at that wavelength. Such a power gives rise to a temperature increase of $0.9 \mathrm{~K}$ in the center of the membrane (see the Supporting Information), indicating that an SNR on the temperature estimation as high as 300 can be achieved. Finally, a calibrated power absorption spectrum is shown in Figure $3 \mathrm{~d}$, where the temperature reached at the center of the membrane throughout the entire spectrum is shown. As can be seen, the temperature increases no more than $0.9 \mathrm{~K}$ compared to room temperature. This ensures the noninvasiveness of the technique, the glass transition temperature of $\mathrm{PVP}^{65}$ being $\sim 448 \mathrm{~K}$.

Figure 4a,b compares the NAM-IR spectra with the ATRFTIR (Perkin-Elmer Spectrum 100) spectra for the PVP sample thicknesses ranging from 20 to $500 \mathrm{~nm}$. For both techniques, the comparison is done with a single-sweep recording. The signal acquisition parameters and postprocessing techniques are shown in the Supporting Information. The ATR-FTIR spectra were acquired at 4 and $2 \mathrm{~cm}^{-1}$ resolution; finer resolutions did not provide any reliable results due to a too low SNR. When ATR-FTIR was used, the three bands of PVP were clearly visible for sample thicknesses higher than 100 $\mathrm{nm}$, but only a very broad peak corresponding to the carbonyl frequency at $1680 \mathrm{~cm}^{-1}$ was observable for thinner samples (Figure 4a). Instead, our NAM-IR spectroscopy reveals all the expected features in the spectra, resolving clearly the three PVP spectroscopic bands down to a $20 \mathrm{~nm}$ sample thickness, both in low and high vacuum (Figure $4 \mathrm{c}$ ).

To compare the performance of the ATR-FTIR and our NAM-IR system, SNRs were calculated on the baseline corrected spectra. The noise was calculated as the standard deviation of the spectra between 1800 and $1953 \mathrm{~cm}^{-1}$, since no light absorption occurs in this range. As a signal for the calculation of the SNR, we analyzed the amplitude of the carbonyl peak around $1680 \mathrm{~cm}^{-1}$. For all the examined thicknesses, the SNRs of NAM-IR (Figure 5a,b) were always at least one order of magnitude higher than the ATR-FTIR values and up to 43 times higher when compared with the high vacuum recorded spectra. It is worth to note that the high vacuum measurements, due to the higher values of the thermal time constants, need a longer settling time of the QCL source for each wavelength to match the longer membrane thermal time constant. The SNR of NAM-IR spectroscopy is nearly independent of the total acquisition time (Figure 5b), as long as the settling time at each IR wavelength is larger than the membrane thermal time constant.

The SNR of the ATR-FTIR spectra depends on the resolution of the acquisitions, ${ }^{66}$ which is typically 4 or 2 $\mathrm{cm}^{-1}$, and the comparisons of the SNRs between the spectra are made, considering such spectral resolutions. Although FTIR-based spectrophotometers increase the SNR of spectra by coarsening the resolution, the spectra acquired at $4 \mathrm{~cm}^{-1}$ on 20 and $40 \mathrm{~nm}$ sample thicknesses did not lead to a remarkable difference regarding the SNR. Considering that the NAM-IR 
a)

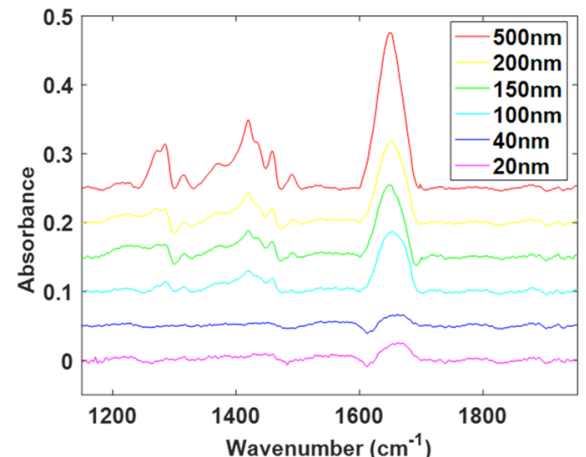

b)

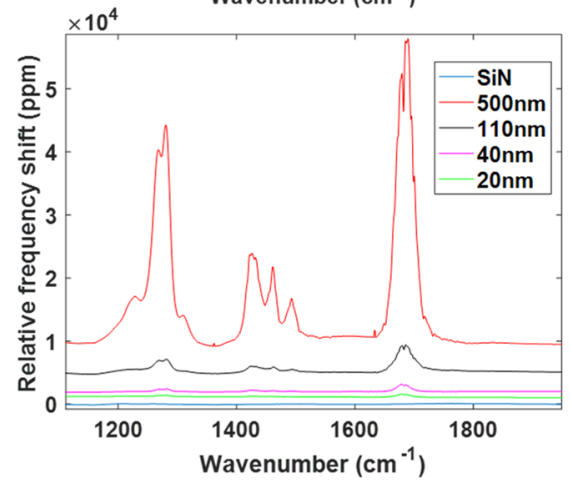

c)

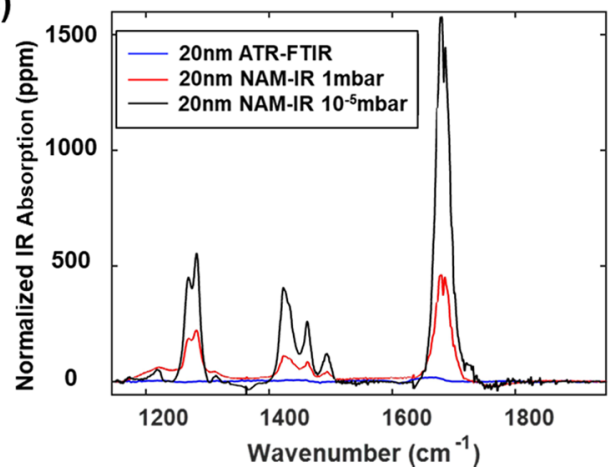

Figure 4. (a) ATR spectra, (b) NAM-IR spectra at 1 mbar, and (c) close up of $20 \mathrm{~nm}$ PVP layer at 1 and $10^{-5}$ mbar and ATR-FTIR spectra. For the sake of comparison, all the spectra were offset, and the NAM-IR spectra are represented as absolute values of the resonance frequency shift.

spectra recorded on a $20 \mathrm{~nm}$-thick PVP layer showed an impressive SNR of 307 in high vacuum, we are confident that this technique is now capable of recording the IR spectrum of a single-molecule PVP layer. In fact, extrapolating the SNR versus thickness relation toward smaller thicknesses (see the Supporting Information) and considering an SNR of 2 as the lowest value needed to clearly recognize a spectral feature of PVP, the limit of detection regarding mass per unit area for NAM-IR is $0.13 \mathrm{fg} / \mu \mathrm{m}^{2}$ (femtogram per square micrometer). This mass per unit area limit corresponds to an equivalent thickness of $160 \mathrm{pm}$ of PVP, approximately corresponding to 4 attomoles of PVP spread over the laser spot area (diameter, $100 \mu \mathrm{m})$. These data strongly indicate that single-molecule layers can be probed with NAM-IR, with very high performances regarding SNR and short acquisition time.

NAM-IR spectroscopy not only outperforms ATR-FTIR in terms of SNR but potentially also in terms of simplicity in the interpretation of the spectrum. In fact, another important feature to bear in mind when comparing our photothermal
NAM-IR with an ATR-FTIR spectrometer regards which vibrational modes can be excited by the IR light in case the sample is a thin film that presents good molecular ordering. ${ }^{13,16,57}$ ATR-FTIR probes the surface sample with the IR beam obliquely oriented to the normal of the sample surface with a nearly fixed angle. Therefore, the IR radiation is probing the perpendicular as well as the horizontal (with respect to the surface) molecular vibrational modes. In this case, the resulting spectra contain both the information about the horizontal as well as the perpendicular modes, calling for precautions in the interpretation of spectra from thin layers ordered at the molecular level. Moreover, the ATR-FTIR spectra further depend on the reflection occurring at the substrate and sample interface. ${ }^{13}$ In fact, when the sampling depth is higher than the sample thickness, the evanescent wave is partially reflected by the substrate, leading to an extinction of the transversal mode and enhancement of the longitudinal ones. ${ }^{13,67}$ Similar artefacts are also present in adsorption-reflectance-based systems. ${ }^{16,20}$ On the other hand, the infrared spectrum obtained by NAM-IR is not influenced by the distortion of the refractive index, which would shift the position of the peaks to lower frequencies. ATR-IR suffers from these artefacts, which need to be corrected through advanced correction algorithms. ${ }^{57}$

Thus, the NAM-IR approach is much more flexible, and the IR light can probe the sample with any angle and any polarization. This flexibility simplifies the interpretation of the spectra for samples where molecules follow a specific orientation on the surface, such as self-assembled monolayers, Langmuir block monolayers, or partially crystallized polymer layers. A spectrum acquired with the IR light impinging the sample surface perpendicularly would contain information only on the vibrational modes that have a component of the dipole moment parallel to the sample surface. Changing the impinging angle of the IR light, the vibrational modes with a dipole moment component orthogonally oriented to the sample surface will also be probed.

These advantages and the much higher SNR for the sub-50 $\mathrm{nm}$ layers make membrane-based NAM-IR a very strong candidate for a new generation of single-molecule layer IR spectroscopy tools.

Membrane resonator-based NAM-IR spectroscopy enabled new possibilities for performing IR spectroscopy on sub-50 nm organic films. The SNR of 307 obtained on a $20 \mathrm{~nm}$ PVP polymer layer, combined with a detection limit in the attomole range, positions membrane-based NAM-IR as a possible technique for a single-molecule layer analysis. The NAM-IR spectroscopy measurements were performed in less than $30 \mathrm{~s}$ on a $20 \mathrm{~nm}$ PVP layer, whereas in using a commercial ATRFTIR technique, the same SNR would be achieved with an acquisition time 43 times longer or an SNR 43 times lower. If we consider the acquisition performed under low vacuum conditions, the SNR is still 25 times higher compared to that of the commercial ATR-FTIR technique.

Moreover, the vibrational modes in ordered organic thin layers can be potentially selectively probed, since the IR light can hit the surface with virtually any angle, overcoming the effects of the interaction of the electric field in the case of reflection of the incident beam at the substrate sample interface. $^{13}$

In terms of the mechanical robustness, the membranes comply better with the experimental conditions typical of the surface functionalization experiments compared to micro- 
a)

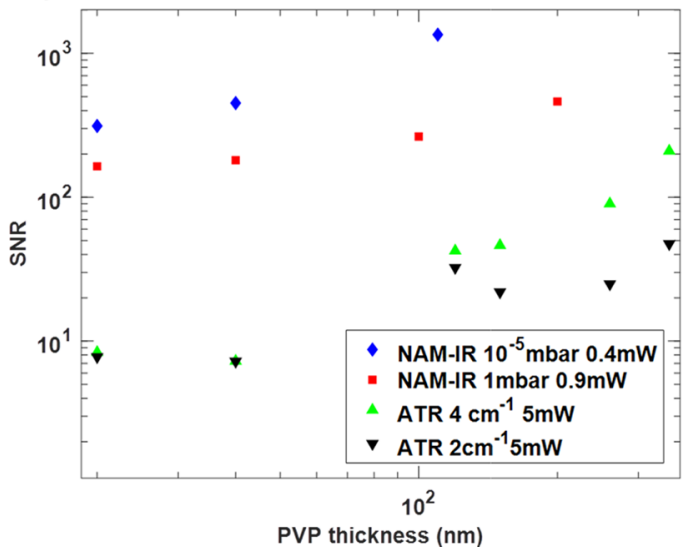

b)

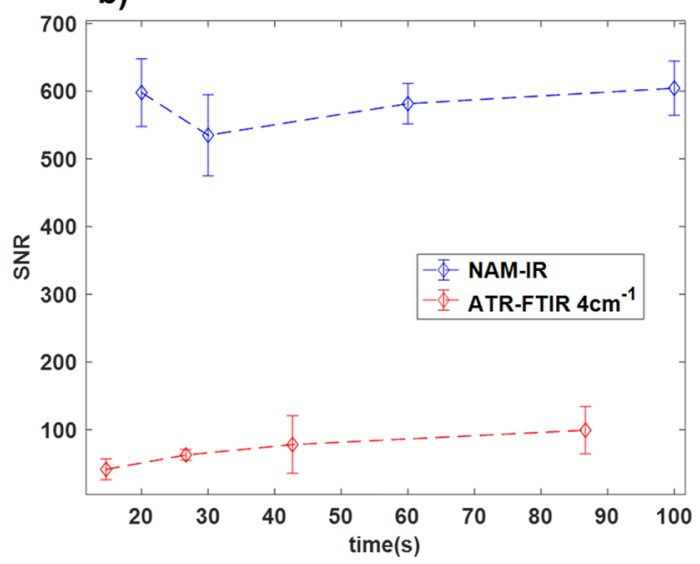

Figure 5. (a) Comparison of SNR between NAM-IR spectroscopy and ATR-FTIR for different thicknesses. (b) SNR of $200 \mathrm{~nm}$ PVP-coated membrane for different acquisition times. The SNR is not sensitive to a prolonged acquisition time; instead, ATR-FTIR spectrophotometer increments the SNR for an increased number of scans.

strings or perforated geometries for airborne particles, allowing the user to prepare the samples in an ample kind of methods. The high capability to dissipate the heat makes the response one order of magnitude faster compared to microstrings. ${ }^{43}$

Finally, the readout of the membrane motion can be integrated using a more advanced design, creating a compact yet extremely sensitive, versatile, and fast IR spectroscopy tool for ultrathin organic layers.

\section{EXPERIMENTAL SECTION}

Membrane Fabrication. The details of the membrane fabrication process are described in the Supporting Information.

Sample Preparation. The polymer solution was prepared by dissolving polyvinylpyrrolidone powder (PVP, SigmaAldrich $\mathrm{Mw}=360,000)$ in an aqueous solution of hydrogen peroxide) $\left(\mathrm{H}_{2} \mathrm{O}_{2}, 30 \% \mathrm{w} / \mathrm{v}\right)$ in two different concentrations (1 and $5 \mathrm{wt} \%)$. The deposition on the membrane was performed by spin coating: the solution $(4 \mathrm{~mL})$ was poured on a silicon wafer containing the membrane chip and then spun at various rotational speeds. The sample was allowed to dry at room temperature and atmospheric pressure. Ellipsometry was used to measure the thickness of the polymer layers. The spinning curves, standard deviations, and details of the sample preparation are provided in the Supporting Information.

NAM-IR Spectra Recording. The NAM-IR signal was recorded through a lock-in scheme. The membrane resonator is placed in a vacuum chamber that guarantees an optical access from the top and from the bottom side of the sensor of the membrane. The resonance frequency of the membrane resonator is probed optically (Polytech MSA-500), and the infrared light is provided by a tunable quantum cascade laser (QCL, Block Engineering 768-1953 $\mathrm{cm}^{-1}$ ) and collimated by a parabolic mirror on the membrane resonator providing a laser spot size of $100 \mu \mathrm{m}$. A lock-in amplifier records the frequency shift. The phase detector bandwidth was $500 \mathrm{~Hz}$ for the $\mathrm{LV}\left(10^{-3}\right.$ mbar) measurement and $200 \mathrm{~Hz}$ for the $\mathrm{HV}$ $\left(10^{-5}\right.$ mbar $)$ measurement, and the sampling rate was set at $3600 \mathrm{~Hz}$. The thermal time constant was determined by irradiating the membrane resonator periodically at a certain wavelength as described elsewhere. ${ }^{58}$ The IR radiation was mechanically chopped providing an irradiation time of $50 \mathrm{~ms}$ for the LV measurement. In $\mathrm{HV}$ mode, the irradiation time was increased to $250 \mathrm{~ms}$ to allow the membrane to reach the steady value of the resonant frequency shift at each cycle.

ATR Spectra Acquisition. The ATR spectra were recorded with Perkin-Elmer Spectrum 100, providing a constant power throughout the spectral range of $5 \mathrm{~mW}$. The spectra were acquired in samples composed of a silicon nitride substrate and a PVP layer, prepared in the same way as the membrane resonator. The PVP layer is placed in tight contact with a single reflection $\mathrm{ZnSe}$ crystal. The gauge force of the crystal was set to its optimal value, achieving the highest signal intensity. For all the spectra, at least three signals were recorded for each sample thickness. A single acquisition spectrum is acquired at the spectral resolution of 2 and $4 \mathrm{~cm}^{-1}$. The amplitude of the acquired spectra is then corrected to compensate the different absorption amplitudes throughout the spectral range using the built-in function of the software.

\section{ASSOCIATED CONTENT}

\section{S Supporting Information}

The Supporting Information is available free of charge on the ACS Publications website at DOI: 10.1021/acsomega.9b00276.

Details on the membrane resonator fabrication, sample preparation, thermal time constant calculations, Allan deviation (noise) measurement, QCL laser power curve, NAM-IR linearity studies, infrared spectroscopy data postprocessing, NAM-IR absorption power, details of the finite element modeling, and limit of the detection calculation (PDF)

\section{AUTHOR INFORMATION}

\section{Corresponding Author}

*E-mail: ancace@nanotech.dtu.dk.

\section{ORCID}

Andrea Casci Ceccacci: 0000-0002-1740-0807

Notes

The authors declare no competing financial interest.

\section{ACKNOWLEDGMENTS}

The research is funded by the Danish National Research Foundation (grant no. DNRF122), the Villum Fonden (grant 
no. 9301), and the European Research Council (grant no. 320535, “HERMES”).

\section{REFERENCES}

(1) Sun, F.; et al. Hierarchical zwitterionic modification of a SERS substrate enables real-time drug monitoring in blood plasma. Nat. Commun. 2016, 7, 13437.

(2) Huang, C. L.; et al. The influence of additives in modulating drug delivery and degradation of PLGA thin films. NPG Asia Mater. 2013, 5, No. e54.

(3) Harrison, R. H.; et al. Modular and Versatile Spatial Functionalization of Tissue Engineering Scaffolds through FiberInitiated Controlled Radical Polymerization. Adv. Funct. Mater. 2015, $25,5748-5757$

(4) Green, M. A. Thin-film solar cells: review of materials, technologies and commercial status. J. Mater. Sci.: Mater. Electron. 2007, 18, 15-19.

(5) Lin, J.; Guo, J.; Liu, C.; Guo, H. Ultrahigh-Performance $\mathrm{Cu}_{2} \mathrm{ZnSnS}_{4}$ Thin Film and Its Application in Microscale Thin-Film Lithium-Ion Battery: Comparison with $\mathrm{SnO}_{2}$. ACS Appl. Mater. Interfaces 2016, 34372.

(6) Ross, C. A.; Berggren, K. K.; Cheng, J. Y.; Jung, Y. S.; Chang, J.B. Three-Dimensional Nanofabrication by Block Copolymer SelfAssembly. Adv. Mater. 2014, 26, 4386-4396.

(7) Hashizume, M.; Murata, Y.; Iijima, K.; Shibata, T. Drug loading and release behaviors of freestanding polysaccharide composite films. Polym. J. 2016, 48, 545-550.

(8) Rahman, A.; et al. Non-native three-dimensional block copolymer morphologies. Nat. Commun. 2016, 7, 13988.

(9) Mishra, R. K.; Datt, M.; Banthia, A. K. Synthesis and characterization of pectin/PVP hydrogel membranes for drug delivery system. AAPS PharmSciTech 2008, 9, 395-403.

(10) Kim, J. H.; Jang, J.; Zin, W.-C. Thickness Dependence of the Glass Transition Temperature in Thin Polymer Films. Langmuir 2001, 2703

(11) Fedorchenko, A. I.; Wang, A.-B.; Cheng, H. H. Thickness dependence of nanofilm elastic modulus. Appl. Phys. Lett. 2009, 94, 152111.

(12) Michell, R. M.; Blaszczyk-Lezak, I.; Mijangos, C.; Müller, A. J. Confinement effects on polymer crystallization: From droplets to alumina nanopores. Polymer 2013, 4059.

(13) Laroche, G.; Fitremann, J.; Gherardi, N. FTIR-ATR spectroscopy in thin film studies: The importance of sampling depth and deposition substrate. Appl. Surf. Sci. 2013, 273, 632-637.

(14) Wang, Y.; et al. Crystallization in the Thin and Ultrathin Films of Poly(ethylene-vinyl acetate) and Linear Low-Density Polyethylene. Macromolecules 2004, 3319.

(15) Ohta, K.; Iwamoto, R. Experimental Proof of the Relation Between Thickness of the Probed Surface Layer and Absorbance in FT-IR/ATR Spectroscopy. Appl. Spectrosc. 1985, 418.

(16) Ramin, M. A.; et al. PM-IRRAS Investigation of Self-Assembled Monolayers Grafted onto $\mathrm{SiO}_{2}$ /Au Substrates. Langmuir 2011, 27, 6076-6084.

(17) Baibarac, M.; et al. SERS spectra of polyaniline thin films deposited on rough $\mathrm{Ag}, \mathrm{Au}$ and $\mathrm{Cu}$. Polymer film thickness and roughness parameter dependence of SERS spectra. Synth. Met. 1998, 96, 63-70.

(18) Kim, J.; Cho, J.; Seidler, P. M.; Kurland, N. E.; Yadavalli, V. K. Investigations of Chemical Modifications of Amino-Terminated Organic Films on Silicon Substrates and Controlled Protein Immobilization. Langmuir 2010, 26, 2599-2608.

(19) Song, S.; Chu, R.; Zhou, J.; Yang, S.; Zhang, J. Formation and Tribology Study of Amide-Containing Stratified Self-Assembled Monolayers: Influences of the Underlayer Structure. J. Phys. Chem. C 2008, 3805 .

(20) Mendelsohn, R.; Mao, G.; Flach, C. R. Infrared reflectionabsorption spectroscopy: principles and applications to lipid-protein interaction in Langmuir films. Biochim. Biophys. Acta, Biomembr. 2010, $1798,788-800$
(21) Jiang, E. Y. Advanced FT-IR Spectroscopy; Thermo Electron Corporation, 2003.

(22) Fan, J.; Trenary, M. Symmetry and the Surface Infrared Selection Rule for the Determination of the Structure of Molecules on Metal Surfaces. Langmuir 1994, 10, 3649-3657.

(23) Adato, R.; Altug, H. In-situ ultra-sensitive infrared absorption spectroscopy of biomolecule interactions in real time with plasmonic nanoantennas. Nat. Commun. 2013, 4, 2154.

(24) Grundmeier, G.; Von Keudell, A.; De los Arcos, T. Fundamentals and Applications of Reflection FTIR Spectroscopy for the Analysis of Plasma Processes at Materials Interfaces. Plasma Processes Polym. 2015, 12, 926-940.

(25) Allara, D. L.; Baca, A.; Pryde, C. A. Distortions of Band Shapes in External Reflection Infrared Spectra of Thin Polymer Films on Metal Substrates. Macromolecules 1978, 11, 1215-1220.

(26) Dazzi, A.; Prater, C. B. AFM-IR: Technology and Applications in Nanoscale Infrared Spectroscopy and Chemical Imaging. Chem. Rev. 2017, 5146.

(27) Xu, X. G.; Rang, M.; Craig, I. M.; Raschke, M. B. Pushing the Sample-Size Limit of Infrared Vibrational Nanospectroscopy: From Monolayer toward Single Molecule Sensitivity. J. Phys. Chem. Lett. 2012, 3, 1836-1841.

(28) Muller, E. A.; Pollard, B.; Bechtel, H. A.; Van Blerkom, P.; Raschke, M. B. Infrared vibrational nano-crystallography and nanoimaging. Sci. Adv. 2016, No. e1601006.

(29) Pollard, B.; Maia, F. C. B.; Raschke, M. B.; Freitas, R. O. Infrared Vibrational Nanospectroscopy by Self-Referenced Interferometry. Nano Lett. 2016, 16, 55-61.

(30) Felts, J. R.; Kjoller, K.; Lo, M.; Prater, C. B.; King, W. P. Nanometer-scale infrared spectroscopy of heterogeneous polymer nanostructures fabricated by tip-based nanofabrication. ACS Nano 2012, 6, 8015-8021.

(31) Bialkowski, S. Photothermal spectroscopy methods for chemical analysis. John Wiley \& Sons, 1996.

(32) Barnes, J. R.; et al. A femtojoule calorimeter using micromechanical sensors. Rev. Sci. Instrum. 1994, 65, 3793.

(33) Barnes, J. R.; Stephenson, R. J.; Welland, M. E.; Gerber, C.; Gimzewski, J. K. Photothermal spectroscopy with femtojoule sensitivity using a micromechanical device. Nature 1994, 372, 79-81.

(34) Vasiliev, A.; et al. On-Chip Mid-Infrared Photothermal Spectroscopy Using Suspended Silicon-on-Insulator Microring Resonators. ACS Sens. 2016, 1301.

(35) Bosco, F. G.; et al. High throughput label-free platform for statistical bio-molecular sensing. Lab Chip 2011, 11, 2411-2416.

(36) Ndieyira, J. W.; et al. Surface-stress sensors for rapid and ultrasensitive detection of active free drugs in human serum. Nat. Nanotechnol. 2014, 9, 225-232.

(37) Patil, S. B.; et al. Decoupling competing surface binding kinetics and reconfiguration of receptor footprint for ultrasensitive stress assays. Nat. Nanotechnol. 2015, 10, 899-907.

(38) Alsteens, D.; et al. Nanomechanical mapping of first binding steps of a virus to animal cells. Nat. Nanotechnol. 2017, 12, 177-183.

(39) Kosaka, P. M.; Pini, V.; Calleja, M.; Tamayo, J. Ultrasensitive detection of HIV-1 p24 antigen by a hybrid nanomechanicaloptoplasmonic platform with potential for detecting HIV-1 at first week after infection. PLoS One 2017, 12, No. e0171899.

(40) Bose, S.; et al. Micromechanical string resonators: Analytical tool for thermal characterization of polymers. ACS Macro Lett. 2014, $3,55-58$.

(41) Jung, N.; Seo, H.; Lee, D.; Ryu, C. Y.; Jeon, S. Nanomechanical Thermal Analysis of the Glass Transition of Polystyrene Using Silicon Cantilevers. Macromolecules 2008, 41, 6873-6875.

(42) Khan, M. F.; et al. Heat capacity measurements of sub-nanoliter volumes of liquids using bimaterial microchannel cantilevers. Appl. Phys. Lett. 2016, 108, 211906.

(43) Larsen, T.; Schmid, S.; Villanueva, L. G.; Boisen, A. Photothermal analysis of individual nanoparticulate samples using micromechanical resonators. ACS Nano 2013, 7, 6188-6193. 
(44) Yamada, S.; Schmid, S.; Larsen, T.; Hansen, O.; Boisen, A. Photothermal Infrared Spectroscopy of Airborne Samples with Mechanical String Resonators. Anal. Chem. 2013, 85, 10531-10535.

(45) Andersen, A. J.; Yamada, S.; Pramodkumar, E. K.; Andresen, T. L.; Boisen, A.; Schmid, S. Nanomechanical IR spectroscopy for fast analysis of liquid-dispersed engineered nanomaterials. Sens. Actuators, B 2016, 233, 667-673.

(46) Ghoraishi, M. S.; Hawk, J. E.; Phani, A.; Khan, M. F.; Thundat, T. Clustering mechanism of ethanol- water mixtures investigated with photothermal microfluidic cantilever deflection spectroscopy. Sci. Rep. 2016, 23966.

(47) Wig, A.; Arakawa, E. T.; Passian, A.; Ferrell, T. L.; Thundat, T. Photothermal spectroscopy of Bacillus anthracis and Bacillus cereus with microcantilevers. Sens. Actuators, B 2006, 114, 206-211.

(48) Biswas, T. S.; et al. Femtogram-scale photothermal spectroscopy of explosive molecules on nanostrings. Anal. Chem. 2014, 86, 11368-11372.

(49) Lee, D.; et al. Plasmonic absorbers with optical cavity for the enhancement of photothermal/opto-calorimetric infrared spectroscopy. Appl. Phys. Lett. 2017, 110, No. 011901.

(50) Pini, V.; et al. How two-dimensional bending can extraordinarily stiffen thin sheets. Sci. Rep. 2016, 6, 29627.

(51) Kurek, M.; et al. Nanomechanical Infrared Spectroscopy with Vibrating Filters for Pharmaceutical Analysis. Angew. Chem. Int. Ed. 2017, 56, 3901-3905.

(52) Marizza, P.; et al. Synthesis and characterization of UV photocrosslinkable hydrogels with poly(N-vinyl-2-pyrrolidone): Determination of the network mesh size distribution. Int. J. Polym. Mater. Polym. Biomater. 2016, 65, 516-525.

(53) Plungpongpan, K.; et al. Preparation of PVP/MHEC Blended Hydrogels via Gamma Irradiation and their Calcium ion Uptaking and Releasing Ability. Energy Procedia 2013, 34, 775-781.

(54) Zhu, X.; Lu, P.; Chen, W.; Dong, J. Studies of UV crosslinked poly(N-vinylpyrrolidone) hydrogels by FTIR, Raman and solid-state NMR spectroscopies. Polymer 2010, 51, 3054-3063.

(55) Prashanthi, K.; Phani, A.; Thundat, T. Photothermal Electrical Resonance Spectroscopy of Physisorbed Molecules on a Nanowire Resonator. Nano Lett. 2015, 15, 5658-5663.

(56) Chae, I.; et al. Standoff Mechanical Resonance Spectroscopy Based on Infrared- Sensitive Hydrogel Microcantilevers. Anal. Chem. 2016, 9678.

(57) Nunn, S.; Nishikida, K. Advanced ATR Correction Algorithm; Thermo Electron Corporation Application Note, 2003.

(58) Miriyala, N.; Khan, M. F.; Thundat, T. Thermomechanical behavior of a bimaterial microchannel cantilever subjected to periodic IR radiation. Sens. Actuators, B 2016, 235, 273-279.

(59) Cleland, A. N.; Roukes, M. L. Noise processes in nanomechanical resonators. J. Appl. Phys. 2002, 2758.

(60) Lam, Y. C.; Joshi, S. C.; Tan, B. K. Thermodynamic characteristics of gelation for methyl-cellulose hydrogels. J. Therm. Anal. Calorim. 2007, 87, 475-482.

(61) Root, S. E.; Alkhadra, M. A.; Rodriquez, D.; Printz, A. D.; Lipomi, D. J. Measuring the Glass Transition Temperature of Conjugated Polymer Films with Ultraviolet-Visible Spectroscopy. Chem. Mater. 2017, 29, 2646-2654.

(62) Lim, A. W.; Löbmann, K.; Grohganz, H.; Rades, T.; Chieng, N. Investigation of physical properties and stability of indomethacincimetidine and naproxen-cimetidine co-amorphous systems prepared by quench cooling, coprecipitation and ball milling. J. Pharm. Pharmacol. 2016, 68, 36-45.

(63) Zuo, B.; et al. Grafting density dominant glass transition of dry polystyrene brushes. Soft Matter 2017, 13, 2426-2436.

(64) Daimon, H.; Okitsu, H.; Kumanotani, J. Glass Transition Behaviors of Random and Block Copolymers and Polymer Blends of styrene and Cyclododecyl Acrylate. I. Glass Transition Temperatures. Polym. J. 1975, 7, 460-466.

(65) Turner, D. T.; Schwartz, A. The glass transition temperature of poly (N- vinyl pyrrolidone) by differential scanning calorimetry. Polymer 1985, 757.
(66) Liu, Q.; Xiao, S. Effects of spectral resolution and signal-tonoise ratio of hyperspectral sensors on retrieving atmospheric parameters. Opt. Lett. 2014, 60.

(67) Dupont, G.; Caquineau, H.; Despax, B.; Berjoan, R.; Dollet, A. Structural properties of N-rich a-Si - N:H films with a low electrontrapping rate. J. Phys. D: Appl. Phys. 1997, 30, 1064-1076. 